\title{
HI observations of nearby galaxies
}

\section{More dwarf galaxies in the southern sky}

\author{
W. K. Huchtmeier ${ }^{1}$, I. D. Karachentsev ${ }^{2}$, and V. E. Karachentseva ${ }^{3}$ \\ 1 Max-Plank-Institut für Radioastronomie, Auf dem Hügel 69, 53121 Bonn, Germany, \\ 2 Special Astrophysical Observatory, Russian Academy of Sciences, N. Arkhyz, KChR, 369167, Russia \\ 3 Astronomical Observatory of Kiev University, Observatorna, 3, $04053 \mathrm{Kiev}$, Ukraine
}

Received 19 June 2001 / Accepted 2 August 2001

\begin{abstract}
In this paper we present $\mathrm{H}$ I observations of nearby galaxies from two more lists of the Karachentsev catalog of candidates for nearby dwarf galaxies in the southern sky. Observations north of declination $-31^{\circ}$ were performed with the 100-m radio telescope at Effelsberg. Data for more southern galaxies were taken from HIPASS (www.atnf.csiro.au/research/multibeam). This sample is a supplement to the list of 94 southern dwarf galaxy candidates $(1998$, A\&AS, 127, 409) located in the same declination range around the known Local Volume galaxies (i.e. galaxies within $10 \mathrm{Mpc}, \mathrm{LV}$ hereafter). We observed 17 galaxies not observed in the previous sample; and all 67 candidate dwarf galaxies from the SERC EJ sky survey (Karachentsev et al. 2000) and 81 objects from a supplementary list of candidate dwarf galaxies (Karachentseva \& Karachentsev 2000). This yields a total of $165(=17+67+81)$ galaxies. Overall we have a detection rate of $48 \%$. The sample of detected galaxies has the following median parameters: radial velocity $V_{\mathrm{LG}}=1127 \mathrm{~km} \mathrm{~s}^{-1}$, H I line width $W_{50}=59 \mathrm{~km} \mathrm{~s}^{-1}$, absolute blue magnitude $M_{\mathrm{BT}}^{0}=-14.4 \mathrm{mag}$, linear diameter $A_{0}=4.0 \mathrm{kpc}$, hydrogen mass-to-luminosity ratio $1.6 M_{\odot} / L_{\odot}$. The sample of known galaxies within the Local Volume increased from 179 in 1979 (Kraan-Korteweg \& Tammann $1979)$ to 387 now. This corresponds to an increase in total luminosity of $22 \%$. The known H I mass in the LV increased by $25 \%$; the relative $\mathrm{H}$ I content $M_{\mathrm{HI}} / L_{\mathrm{B}}$ increased from 0.21 to 0.26 for the whole volume. However we still might have missed half of the dwarf galaxies in the Local Volume.
\end{abstract}

Key words. galaxies: distances and redshifts - radio lines: galaxies

\section{Introduction}

Including the present list of objects, all 601 candidate dwarf galaxies of the Karachentsev survey of the whole sky have been searched for in the Hi line. In the northern sky the sample contains 173 candidates (Huchtmeier et al. 2000a, PaperI) plus 74 objects in the Local Void region (Huchtmeier et al. 2000b, Paper II) and 99 more objects (Karachentsev 2001, Paper III), i.e. 351 galaxies in total. For the southern sky there are 84 galaxies in Paper I plus 4 in Paper II and 2 in Paper III plus 160 in the present paper, i.e. a total of 250 southern objects.

The first step toward a reference sample of nearby galaxies was undertaken by Kraan-Korteweg \& Tammann (1979) who published a list of 179 galaxies with corrected radial velocities $V_{\mathrm{LG}}<500 \mathrm{~km} \mathrm{~s}^{-1}$ with respect

Send offprint requests to: W. K. ,

e-mail: huchtmeier@mpifr-mpg.de

* Table 1 is also, and Table 2 only, available in electronic form at the CDS via anonymous ftp to

cdsarc.u-strasbg.fr (139.79.128.5) or via

http://cdsweb.u-strasbg.fr/cgi-bin/qcat?J/A+A/377/801 to the Local Group centroid. This sample was updated by Huchtmeier \& Richter $(1988,1989)$ and Karachentsev (1994) to reach 215 galaxies. Over the last years the nearby galaxy census was increased by $\mathrm{H}$ I line surveys of the Milky Way (Henning et al. 1998), as well as a "blind" $\mathrm{H} \mathrm{I}$ - survey of the southern sky with the Australian multibeam radio telescope (Kilborn et al. 1999; Banks et al. 1999). In addition to these activities 94 galaxies from the Karachentsev catalog were found to be within a distance of $10 \mathrm{Mpc}$, increasing the LV sample to a total of 387 galaxies. The advantage in investigating a nearby sample is that it is only for nearby galaxies that we have the sensitivity in the optical and radio domain to detect the intrinsically faintest galaxies and hence to study the luminosity function of galaxies and the $\mathrm{H}$ I mass function down to faint and tiny galaxies. The obvious disadvantages of the Local Volume are the facts that we do have an all sky sample, and that the zone of avoidance is a crowded and absorbed area. In addition, the local neutral hydrogen confuses extragalactic Hi emission in the (heliocentric) velocity range $-150 \leq 30$ or $\leq 50 \mathrm{~km} \mathrm{~s}^{-1}$, especially 
for faint HI lines. This only affects part of the Local Group galaxies and the M 81 group, the IC 342/Maffei group and the NGC 6946 group. Even in the Local Volume we are far from being complete, optically we are definitely incomplete for galaxies fainter than $m_{\mathrm{BT}}=16$. A rough estimate shows that we might still miss half the galaxies in the Local Volume.

In this paper we present new HI observations in Sect. 2, and describe the data in Sect. 3 followed by a discussion in Sect. 4.

\section{Observations}

The 100-m radio telescope at Effelsberg has been used in the total power mode (ON-OFF) combining a reference field 5 min earlier in RA or another emission-free field with the on-source position. The dual channel HEMT receiver had a system noise of $30 \mathrm{~K}$. The 1024 channel autocorrelator was split into four bands (each $6.25 \mathrm{MHz}$ wide) of 256 channels each shifted in frequency by $5 \mathrm{MHz}$ with respect to their neighboring band in order to cover a velocity range from -470 to $3970 \mathrm{~km} \mathrm{~s}^{-1}$ with a $1.5 \mathrm{MHz}$ overlap between individual frequency bands. The resulting channel separation was $5.1 \mathrm{~km} \mathrm{~s}^{-1}$ yielding a resolution of $6.2 \mathrm{~km} \mathrm{~s}^{-1}\left(10.2 \mathrm{~km} \mathrm{~s}^{-1}\right.$ after Hanning smoothing). The $\mathrm{H}$ I profiles observed with the $100-\mathrm{m}$ radio telescope are presented in Fig. 1a in order of increasing RA H I profiles for galaxies from "supplementary dwarf galaxy candidates" (Karachentseva \& Karachentsev 2000) are given in Fig. 1b. The half power beamwidth (HPBW) of the Effelsberg telescope at this wavelength is $9.3^{\prime}$.

\section{The data}

Optical data of our galaxies are given in Tables 1a and $1 \mathrm{~b}$ corresponding respectively to Figs. 1a and $1 \mathrm{~b}$; Table 1c corresponds to $\mathrm{HI}$ data extracted from the HIPASS data bank (e.g. Barnes et al. 2001). Their successive columns contain: (1) the running number (kks, kksg, and kk); (2) and (3) equatorial coordinates (epoch 1950.0); (4) and (5) major and minor angular diameter measured on blue plates to the limiting isophote $\sim(26.5-27.0) \mathrm{mag} / \square^{\prime \prime}$ with an accuracy of $\sim 15 \%$; (6) morphological type in the usual designations: $\mathrm{Sph}$ - spheroidal, Ir - irregular, Sm - irregular with signs of spiral structure; (7) mean surface brightness to the limiting isophote in a scale: $\mathrm{H}$ - high $\left(22-23 \mathrm{mag} / \square^{\prime \prime}\right), \mathrm{L}-$ low $\left(\sim 24 \mathrm{mag} / \square^{\prime \prime}\right)$, VL - very low $\left(\sim 25 \mathrm{mag} / \square^{\prime \prime}\right)$, EL extremely low $\left(\sim 26 \mathrm{mag} / \square^{\prime \prime}\right)$; (8) apparent blue magnitude of the galaxy estimated visually by I. Karachentsev (IK) with an accuracy of $0.5 \mathrm{mag}$ based on comparison with other LSB galaxies having photometrically measured total magnitudes or from the NED; (9) reference for the magnitude; (10) galactic extinction $A_{b}$ from Schlegel et al. (1998); (11) comments.

About $64 \%$ of the objects are classified as irregular (Ir or Ir?), $13.5 \%$ as spheroidal (Sph) while all kinds of disk galaxy types and BCD's are to be found among the other galaxies. The majority of all galaxies (90.1\%) are low surface brightness galaxies while about a third of these (35\%) having very low and extremely low surface brightness.

The measured and derived parameters of H I observations are given in Tables $2 \mathrm{a}-2 \mathrm{c}$ for the data sets corresponding to Tables 1a-1c. Table $2 \mathrm{a}$ and part of Table $2 \mathrm{~b}$ display $\mathrm{H}$ I data obtained with the Effelsberg radio telescope. Table $2 \mathrm{c}$ and part of Table $2 \mathrm{~b}$ contain H I data extracted from HIPASS. Their columns contain: (1) the running number; (2) the $\mathrm{H}$ I-flux in $\mathrm{Jy} \mathrm{km} \mathrm{s}^{-1}$; (3 the maximum emission and/or the rms noise in mJy; (4) the mean heliocentric radial velocity and its error; (5) the line widths at the $50 \%$, the $25 \%$, and the $20 \%$ level of the peak emission; (6) the radial velocity with respect to the Local Group centroid with the solar apex parameters: $V_{\mathrm{A}}=316 \mathrm{~km} \mathrm{~s}^{-1}, l_{\mathrm{A}}=93^{\circ}$, $b_{\mathrm{A}}=-4^{\circ}$ (Karachentsev \& Makarov 1996); (7) the integrated absolute magnitude corrected for galactic extinction $A_{b}$ (Schlegel et al. 1998) assuming a kinematic distance $D=V_{\mathrm{LG}} / H_{0}$ with a Hubble constant of $H_{0}=75 \mathrm{~km} \mathrm{~s}^{-1} \mathrm{Mpc}^{-1} ;$ (8) the integrated H I mass-toluminosity ratio in solar units, where

$\left(M_{\mathrm{H} \text { I }} / M_{\odot}\right)=2.35 \times 10^{5} \times D^{2} \times S_{\mathrm{H}_{\mathrm{I}}} ;$

(9) the galaxy "dynamical" mass-to-luminosity ratio in solar units, where the indicative dynamical mass

$M_{\mathrm{dyn}}=0.83 \times 10^{4} \times a \times W_{50}^{2} \times D$

is obtained from the measured angular blue diameter $a$ (Table 1, Col. 4) in arcmin and the Hi line width in $\mathrm{km} \mathrm{s}^{-1}$; here we did not introduce a correction for galaxy inclination because of its uncertainty for irregular galaxies. Therefore the calculated value is a lower limit for the dynamical galaxy mass inside the isophote of $\sim 27 \mathrm{mag} / \square^{\prime \prime}$; (10) comments concerning galaxy membership in groups or cases of confusion with galactic hydrogen emission.

\section{Discussion}

In Papers I to IV we have presented H I observations of galaxies of the whole Karachentsev catalog with an average detection rate of $52 \%$ (values are between $60 \%$ for Paper I and $42 \%$ for Paper II). Most profiles in Figs. 1a and 1b show the typical signature of dwarf galaxies, single-peaked narrow profiles. Only a few wider double-horned profiles are present.

In the following we will discuss the complete data set (Papers I to IV) in order to give an overview of the galaxy population in the Karachentsev catalog. As this catalog is the result of a search for candidate nearby dwarf galaxies, we expect faint galaxies with a small apparent extent. A cut-off of 0.5 arcmin was chosen in order to exclude low surface brightness galaxies at greater distances. In Fig. 2 we present the frequency distribution of the apparent blue diameter of all galaxies (in the $D_{25}$ system) by steps of 0.1 arcmin starting with 0.5 arcmin (the cutoff) for all 

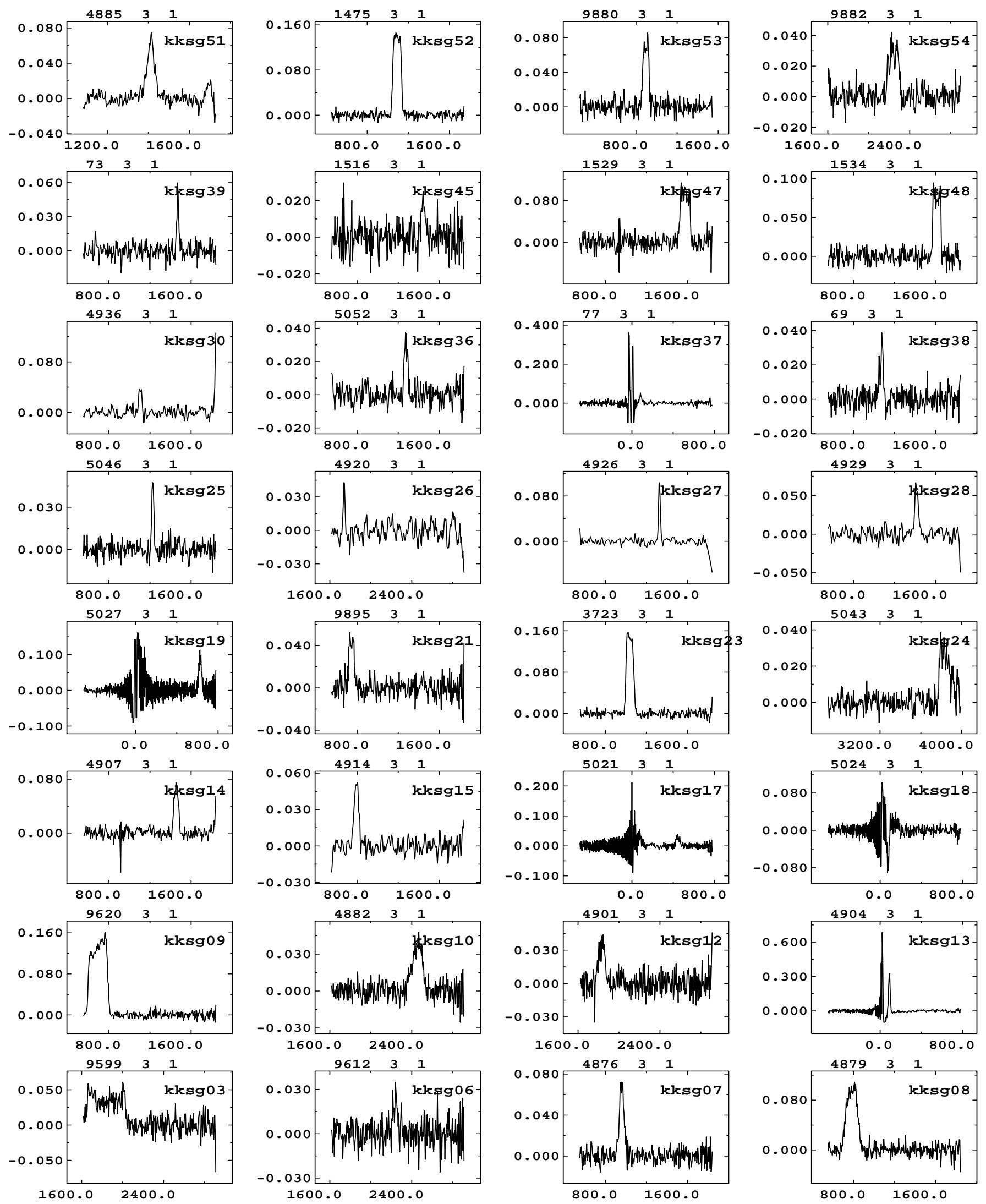

Fig. 1. a) H I profiles for dwarf galaxy candidates in the SERC EJ sky survey observed with the Effelsberg 100-m radio telescope. The profiles are arranged by increasing RA starting at the bottom left corner. The flux scale is in Jy, the heliocentric radial velocity (optical convention) in $\mathrm{km} \mathrm{s}^{-1}$. The velocity resolution of most spectra is $6.2 \mathrm{~km} \mathrm{~s}^{-1}$. The profiles of kksg26, 27, and 30 have been Hanning smoothed (resolution: $10.2 \mathrm{~km} \mathrm{~s}^{-1}$ ). 

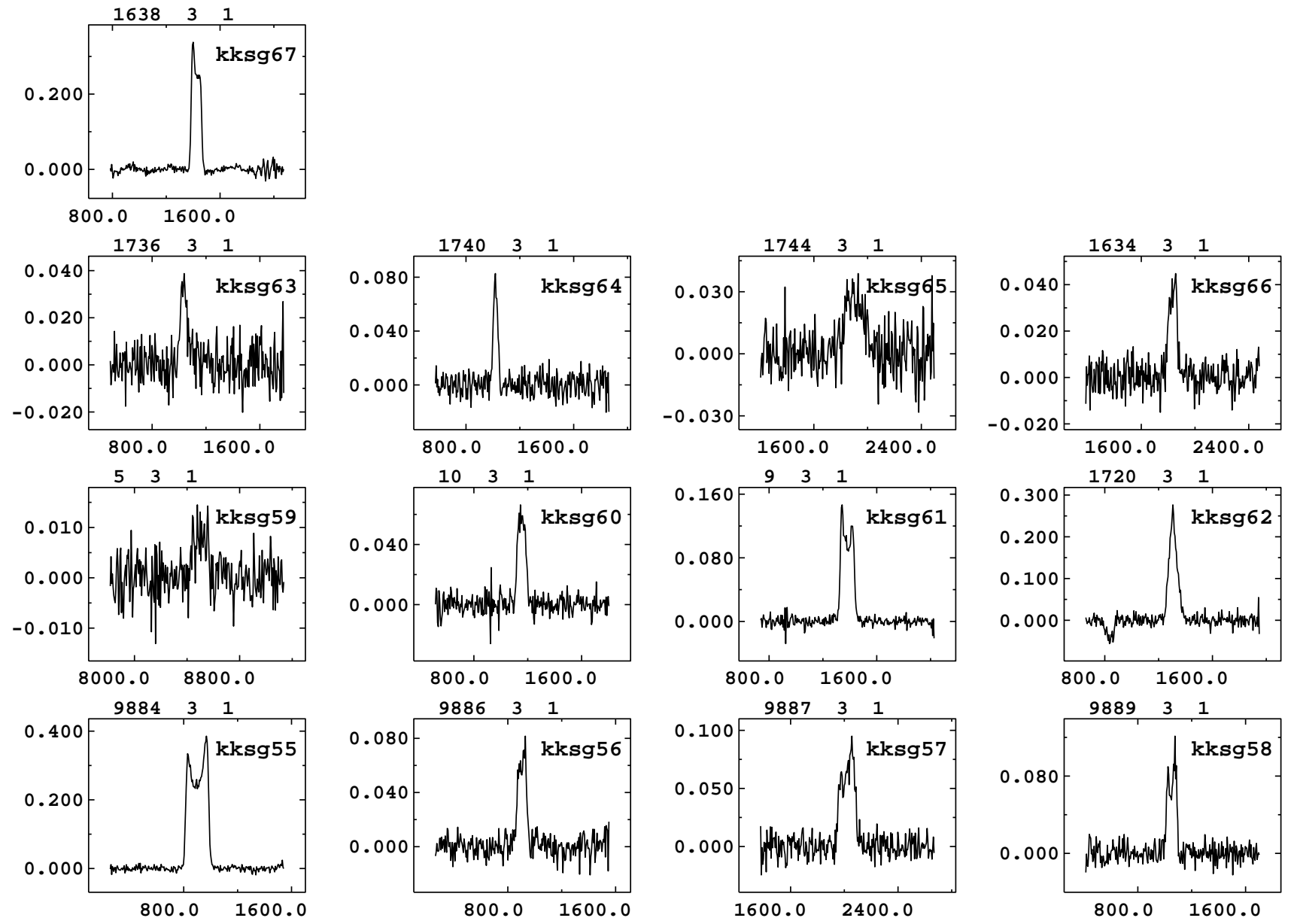

Fig. 1. a) continued.
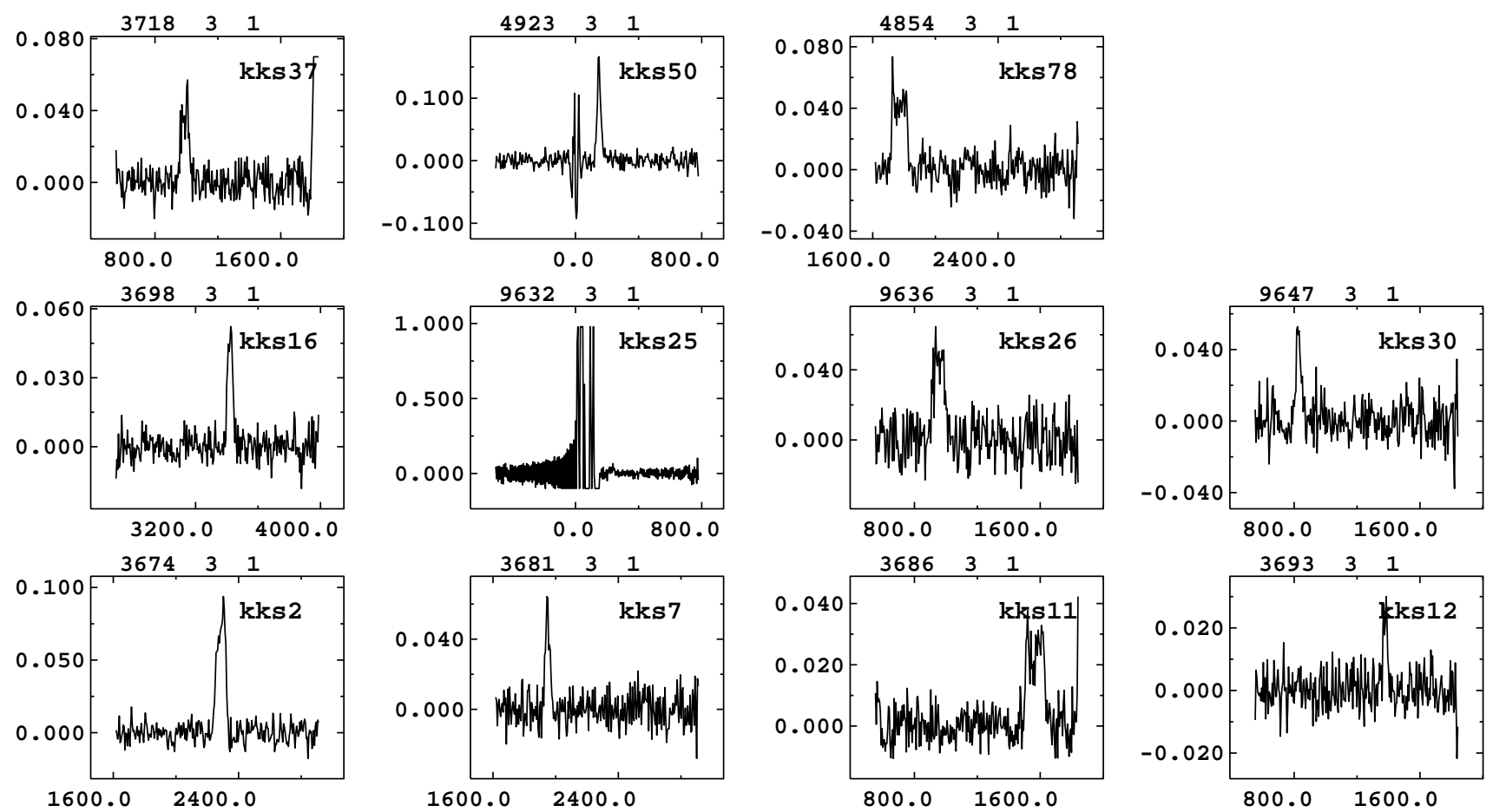

Fig. 1. b) H I profiles for dwarf galaxy candidates from Karachentseva \& Karachentsev (2000) observed with the 100-m radio telescope at Effelsberg. The flux scale is in Jy, the heliocentric radial velocity in $\mathrm{km} \mathrm{s}^{-1}$. The velocity resolution of the spectra is $6.2 \mathrm{~km} \mathrm{~s}^{-1}$. 


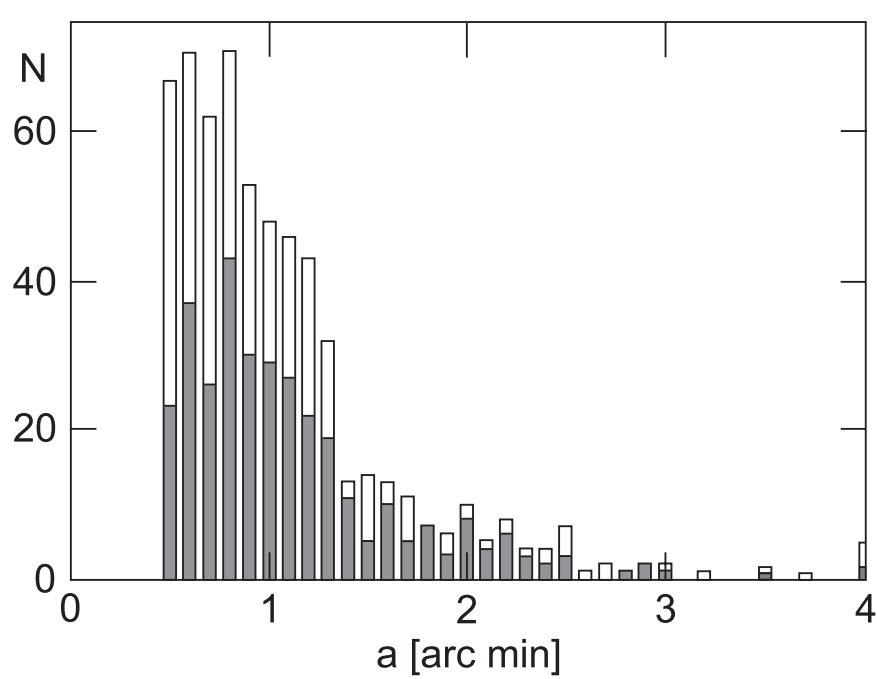

Fig. 2. The distribution of the apparent blue diameters $a_{25}$ binned to 0.1 armin of fall galaxies (Papers I to IV) and the detected galaxies (in grey) is shown. There are 4 galaxies with apparent blue diameters between 4 and 6 arcmin not beyond the scope of this figure.

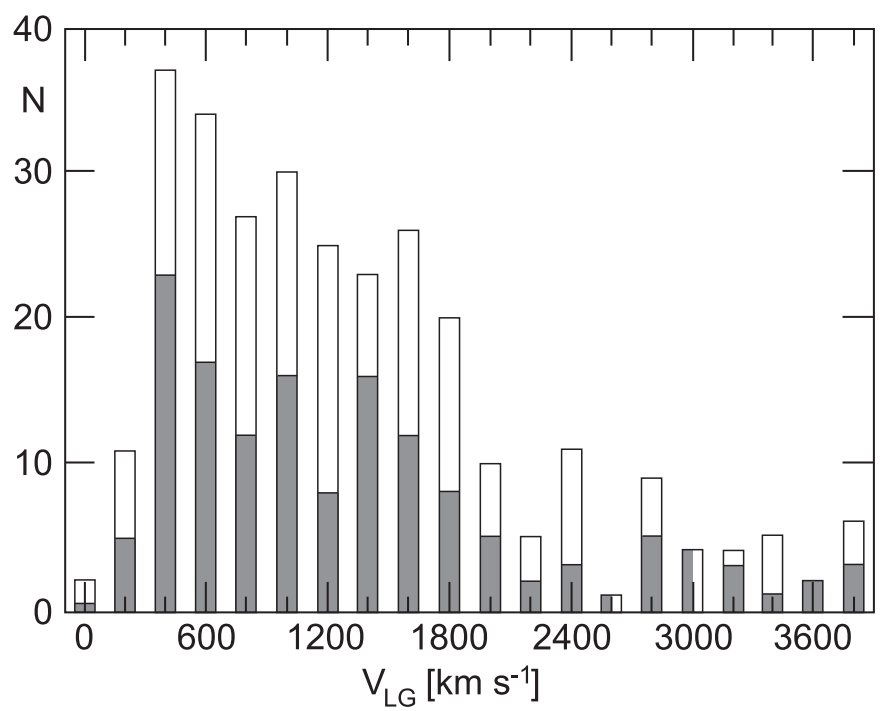

Fig. 3. The radial velocity (corrected for the motion of the Local Group), $V_{\mathrm{LG}}$, distribution is given for all detected galaxies and for the detected galaxies of Paper I (in grey) is given here, binned into velocity intervals of $200 \mathrm{~km} \mathrm{~s}^{-1}$ width.

galaxies and for the detected galaxies. The majority of the galaxies in this sample has small angular extent. More than $50 \%$ of the galaxies are smaller than 1 arcmin, a trend already found in Paper I.

As all other parameters are available for the detected galaxies we will only consider from now on the detected galaxies. The distribution of their radial velocities corrected for the motion of the Local Group, $V_{\mathrm{LG}}$, is given in Fig. 3 for the whole sample and for Paper I binned into velocity intervall of $200 \mathrm{~km} \mathrm{~s}^{-1}$. The similarity of both samples is obvious. Most galaxies are relatively nearby; they belong to the Local supercluster. Next we will consider the distribution of apparent blue magnitudes. All detected

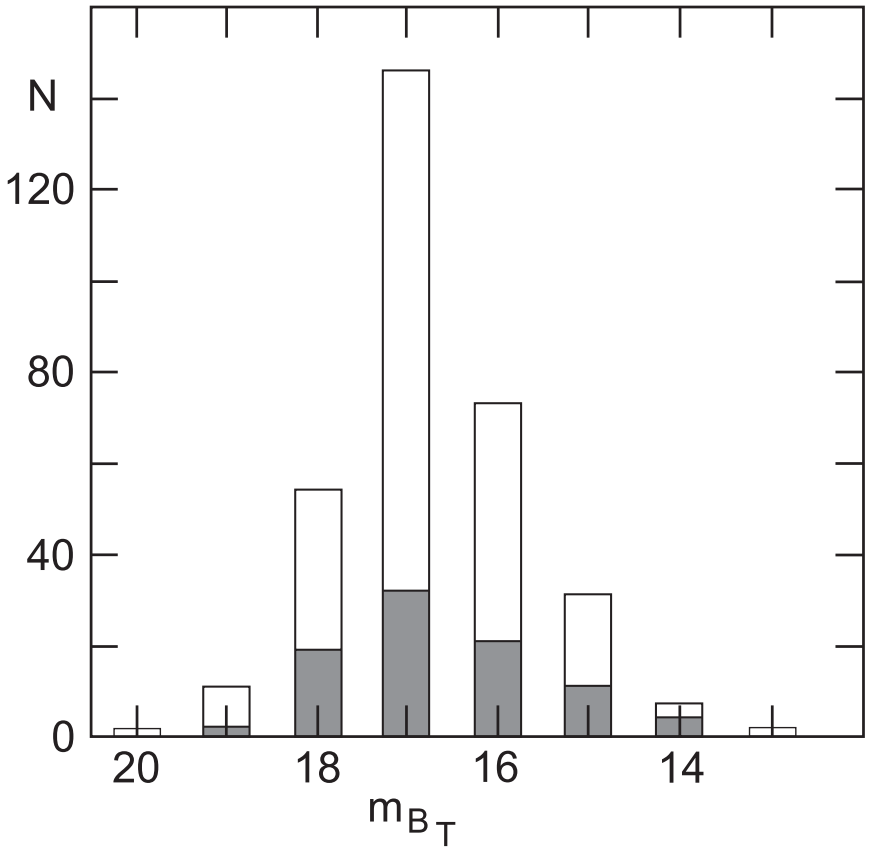

Fig. 4. The distribution of the apparent blue magnitude $m_{\mathrm{BT}}$ binned to 0.5 mag. of all detected galaxies (Papers I to IV) and the detected galaxies for the present data (Paper IV) is shown (in grey).

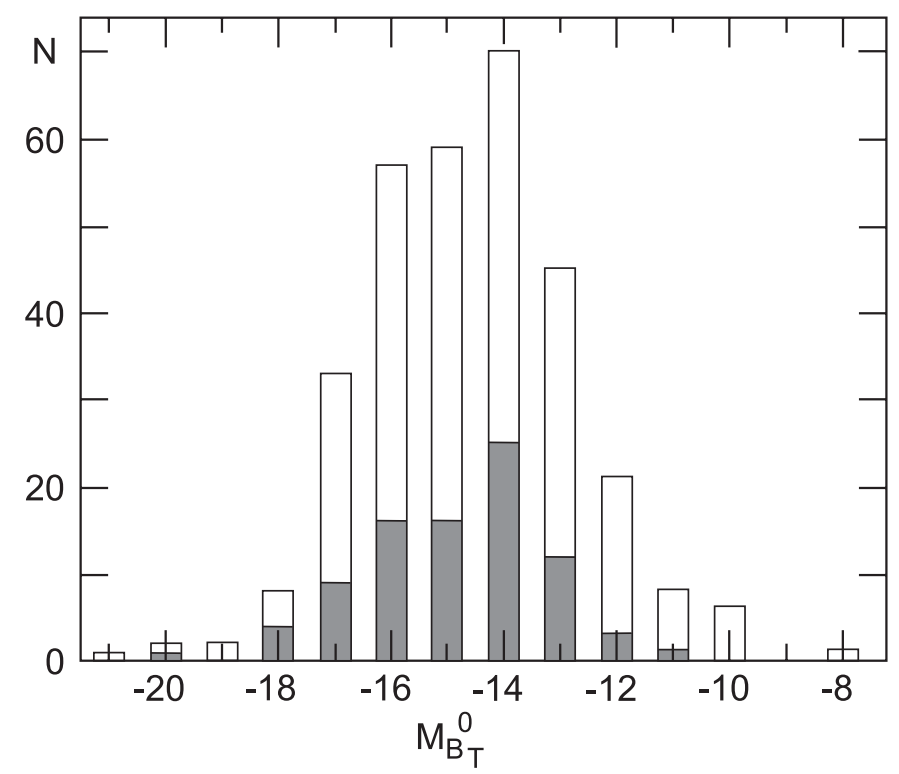

Fig. 5. The distribution of the absolute blue magnitude $M_{\mathrm{BT}}^{0}$ binned to 0.5 mag. of all detected galaxies (Papers I to IV) and the detected galaxies of the present paper (in grey) is shown.

galaxies fall within the range of apparent blue magnitude, $m_{\mathrm{BT}}$, between $13 \mathrm{~m}$ and $20 \mathrm{~m}$ (Fig. 4). The distribution of apparent blue magnitude peaks around $m_{\mathrm{BT}}=+17$ and falls down to fainter objects. This obviously is a sign of incompleteness but the cut-off at 0.5 arcmin diameter also reduces the number of faint galaxies.

The dwarfish character of the Karachentsev galaxies becomes evident when considering luminosity and size. We display in Fig. 5 the distribution of the galaxies in 
Table 1. a) List of new dwarf galaxy candidates found in the SERC EJ zone.

\begin{tabular}{|c|c|c|c|c|c|c|c|c|c|c|}
\hline kksg & \multicolumn{2}{|c|}{ RA (1950.0) Dec } & $a$ & $b$ & Type & S.B. & $B_{\mathrm{t}}$ & Ref.(B) & $A_{b}$ & Notes \\
\hline & $\mathrm{h} \mathrm{m} \mathrm{s}$ & & arcm & & & & & & & \\
\hline 1 & 2 & 3 & 4 & 5 & 6 & 7 & 8 & 9 & 10 & 11 \\
\hline 1 & 002338.5 & -111950 & 5.0 & 4.3 & Sph & VL & & & 0.12 & Cetus dw, LG member \\
\hline 2 & 003744.6 & -180604 & 0.9 & 0.6 & $\mathrm{Sph} / \mathrm{Ir}$ & $\mathrm{L}$ & & & 0.08 & \\
\hline 3 & 013216.7 & -073638 & 0.6 & 0.4 & Ir & $\mathrm{L}$ & 17.4 & IK & 0.14 & NGC $6155^{\prime} \mathrm{NE}$ \\
\hline 4 & 023919.2 & -083654 & 1.2 & 1.1 & Sph & $\mathrm{L}$ & & & 0.10 & in NGC 1052 gr.? \\
\hline 5 & 024703.0 & -132506 & 1.0 & 0.5 & Ir & $\mathrm{L}$ & & & 0.12 & \\
\hline 6 & 042759.2 & -141856 & 0.9 & 0.4 & Ir & $\mathrm{L}$ & 17.7 & IK & 0.64 & \\
\hline 7 & 055702.8 & -130528 & 0.8 & 0.2 & Ir & $\mathrm{L}$ & 17.7 & IK & 2.20 & \\
\hline 8 & 064215.2 & -175251 & 1.8 & 0.3 & $\operatorname{Ir}$ & $\mathrm{H}$ & 15.13 & NED & 1.79 & \\
\hline 9 & 064444.3 & -175310 & 0.5 & 0.35 & Epec & $\mathrm{H}$ & 17.2 & IK & 1.91 & NGC $228322^{\prime} \mathrm{S}$ \\
\hline 10 & 071028.9 & -074412 & 1.0 & 0.25 & Ir & $\mathrm{L}$ & 18.5 & IK & 3.38 & \\
\hline 11 & 072841.0 & -005204 & 0.7 & 0.4 & Ir & $\mathrm{L}$ & & & 0.47 & \\
\hline 12 & 085105.8 & -174742 & 0.5 & 0.4 & Ir & $\mathrm{L}$ & 17.4 & IK & 0.80 & \\
\hline 13 & 093305.4 & -160634 & 1.2 & 0.2 & Ir & VL & 18.2 & IK & 0.24 & \\
\hline 14 & 093755.0 & -033930 & 1.1 & 1.0 & Ir & $\mathrm{L}$ & 16.6 & IK & 0.23 & \\
\hline 15 & 095240.9 & -060200 & 1.3 & 0.3 & Ir? & $\mathrm{H}$ & 15.14 & NED & 0.18 & comp. N $3115 ?$ \\
\hline 16 & 095718.2 & -090639 & 0.9 & 0.7 & Ir? & VL & & & 0.31 & comp. N $3115 ?$ \\
\hline 17 & 095909.4 & -080027 & 1.4 & 0.25 & Ir & $\mathrm{H}$ & 15.5 & NED & 0.26 & comp. N $3115 ?$ \\
\hline 18 & 100312.3 & -074416 & 1.7 & 1.4 & S0pec & $\mathrm{H}$ & 13.84 & NED & 0.23 & \\
\hline 19 & 102200.5 & -121043 & 0.7 & 0.6 & Ir & $\mathrm{L}$ & 17.9 & IK & 0.30 & \\
\hline 20 & 110206.3 & +001940 & 0.4 & 0.4 & $\mathrm{Ir} / \mathrm{Sph}$ & $\mathrm{L}$ & & & 0.16 & comp. N $3521 ?$ \\
\hline 21 & 110301.7 & -013536 & 1.7 & 0.8 & $\mathrm{Ir}$ & $\mathrm{L}$ & 17.3 & IK & 0.24 & UGC 6145 , comp. N3521? \\
\hline 22 & 110334.5 & -011036 & 0.5 & 0.4 & Ir? & $\mathrm{L}$ & & & 0.20 & comp. N $3521 ?$ \\
\hline 23 & 110342.2 & -140804 & 3.5 & 1.2 & Ir & $\mathrm{L}$ & 15.8 & IK & 0.23 & \\
\hline 24 & 111120.3 & -031531 & 0.5 & 0.4 & Ir & $\mathrm{L}$ & 17.4 & IK & 0.25 & \\
\hline 25 & 114245.5 & -165946 & 1.2 & 0.7 & Ir & VL & 18.2 & IK & 0.17 & \\
\hline 26 & 115118.3 & -144451 & 1.8 & 1.1 & Ir & VL & 17.8 & IK & 0.17 & \\
\hline 27 & 121930.9 & -093123 & 0.8 & 0.3 & Ir & $\mathrm{L}$ & 17.7 & IK & 0.19 & \\
\hline 28 & 122122.0 & -144034 & 1.2 & 1.0 & Ir & EL & 18.6 & IK & 0.25 & \\
\hline 29 & 123438.4 & -101321 & 1.4 & 0.6 & Ir & $\mathrm{L}$ & & & 0.12 & comp. N $4594 ?$ \\
\hline 30 & 123500.5 & -083532 & 1.0 & 0.5 & Ir & $\mathrm{L}$ & 17.3 & IK & 0.14 & comp. N $4594 ?$ \\
\hline 31 & 123558.0 & -101256 & 0.8 & 0.7 & Sph & VL & & & 0.13 & comp. N $4594 ?$ \\
\hline 32 & 123718.3 & -112835 & 0.6 & 0.5 & Sph & EL & & & 0.21 & comp. N $4594 ?$ \\
\hline 33 & 123732.7 & -120525 & 0.4 & 0.4 & Sph & VL & & & 0.20 & comp. N $4594 ?$ \\
\hline 34 & 123842.7 & -113912 & 0.8 & 0.7 & Sph & VL & & & 0.23 & comp. N $4594 ?$ \\
\hline 35 & 123958.6 & -144002 & 0.4 & 0.4 & Ir & EL & & & 0.22 & \\
\hline 36 & 124402.8 & -034810 & 0.9 & 0.65 & Ir & $\mathrm{L}$ & 17.4 & IK & 0.10 & \\
\hline 37 & 124524.2 & -122300 & 0.6 & 0.5 & Sph? & VL & 18.7 & IK & 0.22 & \\
\hline 38 & 125056.6 & -053925 & 1.2 & 0.5 & Ir & $\mathrm{L}$ & 17.0 & $\mathrm{IK}$ & 0.15 & \\
\hline 39 & 125106.5 & -054846 & 0.8 & 0.6 & Sph & EL & 18.5 & IK & 0.17 & \\
\hline 40 & 125715.7 & -135104 & 1.0 & 0.6 & Ir & VL & & & 0.20 & \\
\hline 41 & 130307.6 & -072929 & 1.4 & 1.1 & Ir & $\mathrm{L}$ & & & 0.20 & comp. N $4948 ?$ \\
\hline 42 & 130342.7 & -074931 & 1.2 & 0.9 & Ir & VL & & & 0.20 & comp. N $4948 ?$ \\
\hline 43 & 130639.6 & -170555 & 0.7 & 0.5 & Ir? & EL & & & 0.31 & \\
\hline 44 & 133023.5 & -120020 & 0.5 & 0.3 & Ir & $\mathrm{L}$ & & & 0.26 & \\
\hline 45 & 141106.0 & -015712 & 0.6 & 0.4 & Sph? & $\mathrm{L}$ & 17.5 & IK & 0.29 & \\
\hline
\end{tabular}


Table 1. a) continued.

\begin{tabular}{|c|c|c|c|c|c|c|c|c|c|c|}
\hline kksg & \multicolumn{2}{|c|}{ RA (1950.0) Dec } & $a$ & $b$ & Type & S.B. & $B_{\mathrm{t}}$ & Ref.(B) & $A_{b}$ & Notes \\
\hline \multicolumn{3}{|c|}{$\mathrm{h} \mathrm{m} \mathrm{s}$} & $\operatorname{arcm}$ & & & & & & & \\
\hline 1 & 2 & 3 & 4 & 5 & 6 & 7 & 8 & 9 & 10 & 11 \\
\hline 46 & 142547.0 & -084155 & 0.5 & 0.5 & Ir & $\mathrm{L}$ & & & 0.25 & \\
\hline 47 & 143238.5 & -165643 & 1.8 & 0.9 & Ir & $\mathrm{L}$ & 17.7 & IK & 0.33 & \\
\hline 48 & 160302.4 & -042616 & 1.3 & 1.0 & Ir & $\mathrm{L}$ & 18.2 & IK & 1.19 & \\
\hline 49 & 164045.7 & -075800 & 1.1 & 0.4 & Ir & $\mathrm{L}$ & & & 1.72 & \\
\hline 50 & 173900.1 & -043559 & 1.3 & 0.5 & $\mathrm{Ir}$ & $\mathrm{L}$ & & & 3.08 & \\
\hline 51 & 023128.0 & -063444 & 0.8 & 0.4 & Ir & $\mathrm{L}$ & 15.4 & IK & 0.11 & \\
\hline 52 & 024650.9 & -025143 & 0.9 & 0.5 & $\mathrm{Ir} / \mathrm{SB}$ & $\mathrm{L}$ & 16.9 & IK & 0.22 & \\
\hline 53 & 040639.3 & -084528 & 1.0 & 0.6 & $\mathrm{Ir} / \mathrm{S}$ & $\mathrm{L}$ & 17.3 & IK & 0.23 & \\
\hline 54 & 053936.7 & -123509 & 1.0 & 0.4 & $\mathrm{Ir} / \mathrm{Sm}$ & $\mathrm{L}$ & 15.4 & IK & 0.73 & \\
\hline 55 & 054755.8 & -101845 & 2.0 & 0.9 & $\mathrm{SB} / \mathrm{Ir}$ & $\mathrm{L}$ & 17.4 & IK & 3.57 & \\
\hline 56 & 054944.0 & -110905 & 0.9 & 0.8 & $\mathrm{Sph} / \mathrm{Ir}$ & VL & 15.5 & NED & 2.90 & pair w.No55 \\
\hline 57 & 084104.4 & -171215 & 1.8 & 0.5 & $\operatorname{Ir}$ & $\mathrm{L}$ & 16.1 & IK & 0.32 & \\
\hline 58 & 105029.1 & +024533 & 1.5 & 0.5 & Ir & $\mathrm{L}$ & 17.4 & NED & 0.16 & CGCG08-043 at $8.8^{\prime}$ \\
\hline 59 & 111013.9 & -001739 & 0.5 & 0.4 & Ir & $\mathrm{L}$ & 17.8 & NED & 0.17 & \\
\hline 60 & 122035.1 & -134005 & 1.4 & 0.9 & Ir & $\mathrm{L}$ & 16.2 & IK & 0.21 & \\
\hline 61 & 124218.7 & -085115 & 4.0 & 1.8 & $\mathrm{Sm}$ & $\mathrm{L}$ & 15.1 & IK & 0.16 & UGCA295 \\
\hline 62 & 125629.6 & -115731 & 1.2 & 0.8 & Ir & $\mathrm{L}$ & 16.6 & IK & 0.18 & \\
\hline 63 & 132343.5 & +024306 & 0.8 & 0.4 & Ir & $\mathrm{L}$ & 16.8 & NED & 0.10 & \\
\hline 64 & 132539.4 & +023220 & 0.8 & 0.45 & Ir & $\mathrm{L}$ & 16.3 & NED & 0.12 & \\
\hline 65 & 141147.0 & -024759 & 0.8 & 0.25 & Ir & $\mathrm{L}$ & 15.8 & NED & 0.30 & \\
\hline 66 & 143120.7 & +014214 & 1.0 & 0.2 & Ir & $\mathrm{L}$ & 17.4 & NED & 0.20 & \\
\hline 67 & 200641.4 & -062605 & 2.2 & 1.4 & S & $\mathrm{L}$ & 15.3 & IK & 0.34 & \\
\hline
\end{tabular}

absolute blue magnitude $M_{\mathrm{BT}}^{0}$ binned into magnitude intervals of 1 magnitude. Apart from a few bright galaxies all objects are fainter than $M_{\mathrm{BT}}^{0}=-18$, i.e. dwarfs. More than half of the detected galaxies are fainter than $M_{\mathrm{BT}}^{0}=-15$. The distribution of galaxies from Paper IV is similar to that for the whole sample. In Fig. 6 the size distribution of the galaxies in linear extent, $A_{0}[\mathrm{kpc}]$, in the de Vaucouleurs $D_{25}$ system, is given for the whole sample and for Paper I. Over $50 \%$ of the galaxies are smaller than $3.5 \mathrm{kpc}$, as in Paper I. The indicative H I surface density is derived by dividing the HI mass by the surface of the galaxy $\left(a^{2} 2.3^{2} \pi\right)$, assuming the $\mathrm{H}$ I extent to be 2.3 times the optical extent, $r_{\mathrm{HI}}=2.3 \times r_{25}$ (Salpeter \& Hoffman 1996). The dynamical mass has been calculated without inclination correction because of the large uncertainty of the inclinations of dwarf galaxies.

Our sample of galaxies (Papers I to IV) may be described by the following parameters (median values):

- apparent blue diameter $a$ : 0.9 arcmin,

- apparent blue magnitude $m_{\mathrm{BT}}=16.22$,

- half power line widths $W_{50}=51 \mathrm{~km} \mathrm{~s}^{-1}$,

- linear diameter $A_{0}=3.2 \mathrm{kpc}$,

- absolute blue magnitude $M_{\mathrm{BT}}^{0}=-14.8$,

- mass of neutral atomic hydrogen $M_{\mathrm{H} \text { I }}=1.5 \times 10^{8} M_{\odot}$,

- relative $\mathrm{H}$ I content $M_{\mathrm{H}} / L_{\mathrm{B}}=2.8$,
- H I surface density $\sigma_{\mathrm{H}}=3.4 M_{\odot} / \mathrm{pc}^{2}$,

- dynamical mass (not corrected for inclination) $M_{\mathrm{dyn}}=$ $3.6 \times 10^{8} M_{\odot}$,

- mass-to-luminosity ratio $M_{\mathrm{dyn}} / L_{\mathrm{B}}=2.4$.

Recently Eder \& Schombert (2000) presented H I data for gas rich dwarf galaxies selected from the POSS II survey and observed with the 305-m Arecibo radio telescope. Their sample has about the same median $M_{\mathrm{H}} / \mathrm{L}_{\mathrm{B}}$ ratio, 2.0 compared to 2.8 for our sample, but their mean H I mass $\left(8 \times 10^{8} M_{\odot}\right)$ exceeds the median of our sample by a factor 5 to 6 ; the dynamical masses for their sample are much higher than for our sample. Therefore, they observed a more distant sample of larger and more massive galaxies.

Eder \& Schombert (2000) used estimated H I radii for their sample of low surface brightness (LSB) galaxies and derived an average $\mathrm{H}$ I surface density range from 0.6 to $20 M_{\odot} / \mathrm{pc}^{2}$, all well below the critical threshold for star formation which is in the range 5 to $30 M_{\odot} / \mathrm{pc}^{2}$ (Kennicutt 1998). This is true for our sample, too, where the median values for the $\mathrm{HI}$ surface density decrease from 4.1 (to 3.4 to 2.7 ) to $2.6 M_{\odot} / \mathrm{pc}^{2}$ with decreasing surface brightness (from high to low to very low to extremely low SB). This decrease is in agreement with Paper I and with de Blok (1997) who compares the H I surface density 
Table 1. b) List of new dwarf galaxy candidates found in the southern sky.

\begin{tabular}{|c|c|c|c|c|c|c|c|c|c|c|}
\hline $\mathrm{kks}$ & \multicolumn{2}{|c|}{ RA (1950.0) Dec } & $a$ & $b$ & Type & S.B. & $B_{\mathrm{t}}$ & Ref.(B) & $A_{b}$ & Notes \\
\hline & $\mathrm{h} \mathrm{m} \mathrm{s}$ & & $\operatorname{arc}$ & $\min$ & & & & & & \\
\hline 1 & 2 & 3 & 4 & 5 & 6 & 7 & 8 & 9 & 10 & 11 \\
\hline 1 & 021245.1 & -322631 & 0.80 & 0.70 & Ir & LV & 18.0: & IK & 0.09 & \\
\hline 2 & 022338.7 & -195507 & 1.30 & 0.60 & Ir & $\mathrm{L}$ & 16.23 & NED & 0.14 & \\
\hline 3 & 022423.1 & -734416 & 2.50 & 1.00 & Sph? & $\mathrm{VL}$ & 16.0: & IK & 0.22 & \\
\hline 4 & 031206.1 & -662722 & 2.0: & 1.0: & Ir & EL & 17.8: & IK & 0.14 & comp. to NGC 1313 \\
\hline 5 & 031212.4 & -381042 & 1.20 & 0.70 & Sph & $\mathrm{L}$ & 17.18 & NED & 0.07 & \\
\hline 6 & 031347.5 & -663432 & $3.2:$ & 1.4: & Ir & EL & 17.0: & IK & 0.24 & comp. to NGC 1313 \\
\hline 7 & 031528.0 & -250530 & 1.20 & 0.60 & Ir & $\mathrm{L}$ & 17.2 & IK & 0.05 & \\
\hline 8 & 031937.2 & -421040 & 1.10 & 0.90 & Ir & $\mathrm{L}$ & 16.37 & NED & 0.05 & \\
\hline 9 & 033336.1 & -513711 & 1.3: & 1.0: & Ir & $\mathrm{VL}$ & 16.24 & NED & 0.06 & \\
\hline 10 & 033733.1 & -315712 & 1.20 & 0.70 & Ir & $\mathrm{L}$ & 16.9 & IK & 0.04 & \\
\hline 11 & 034008.9 & -225454 & 1.90 & 1.40 & S & $\mathrm{VL}$ & 14.92 & NED & 0.08 & projected on E482-36 \\
\hline 12 & 034149.2 & -250448 & 1.0: & $0.7:$ & Ir & $\mathrm{L}$ & 17.49 & NED & 0.06 & \\
\hline 13 & 034712.8 & -483418 & 1.10 & 0.80 & Ir & $\mathrm{L}$ & 16.39 & NED & 0.04 & \\
\hline 14 & 052623.6 & -633616 & 0.70 & 0.55 & Ir & EL & 17.8 & IK & 0.22 & near NGC 1947 \\
\hline 15 & 052645.5 & -631641 & 1.70 & 0.80 & Ir? & $\mathrm{VL}$ & 16.81 & NED & 0.24 & near NGC 1947 \\
\hline 16 & 053010.8 & -174354 & 0.70 & 0.40 & Ir & $\mathrm{L}$ & 17.8 & IK & 0.32 & \\
\hline 17 & 053213.0 & -615749 & 0.95 & 0.65 & Ir & $\mathrm{VL}$ & 17.4 & IK & 0.26 & \\
\hline 18 & 060355.2 & -330433 & 3.5: & $2.5:$ & Ir? & $\mathrm{L}$ & 13.58 & NED & 0.19 & \\
\hline 19 & 061506.3 & -574227 & 1.40 & 1.00 & Ir & $\mathrm{L}$ & 15.85 & NED & 0.17 & ESO 121-G020 \\
\hline 20 & 070121.5 & -445930 & 1.10 & 0.50 & Ir & $\mathrm{L}$ & 16.19 & NED & 0.41 & \\
\hline 21 & 071452.7 & -400021 & 1.30 & 0.40 & Ir & $\mathrm{L}$ & 16.32 & NED & 1.17 & \\
\hline 22 & 073104.6 & -352250 & 1.20 & 0.80 & Ir & $\mathrm{VL}$ & 16.7 & IK & 1.94 & \\
\hline 23 & 074213.9 & -450145 & 0.90 & 0.90 & Sph? & EL & 17.5 & IK & 1.49 & \\
\hline 24 & 075014.8 & -551922 & 1.10 & 0.90 & Ir & $\mathrm{L}$ & 17.3 & IK & 0.89 & \\
\hline 25 & 075433.4 & -260703 & 0.50 & 0.40 & Ir & $\mathrm{VL}$ & 17.7 & IK & 1.45 & \\
\hline 26 & 075543.5 & -264400 & 0.80 & 0.35 & Ir & $\mathrm{VL}$ & 18.0 & IK & 2.67 & \\
\hline 27 & 084439.8 & -213643 & 0.80 & 0.60 & Ir & $\mathrm{L}$ & 17.4 & IK & 0.74 & \\
\hline 28 & 085239.5 & -323734 & 1.20 & 0.50 & Ir & $\mathrm{VL}$ & 17.42 & NED & 1.36 & \\
\hline 29 & 090851.2 & -634127 & 0.75 & 0.45 & Ir & $\mathrm{VL}$ & 17.5 & IK & 0.89 & \\
\hline 30 & 092051.7 & -195710 & 0.60 & 0.40 & $\operatorname{Im}$ & $\mathrm{H}$ & 15.53 & NED & 0.27 & \\
\hline 31 & 093038.2 & -330120 & 1.3: & $0.7:$ & Ir & $\mathrm{L}$ & 16.4 & IK & 0.58 & \\
\hline 32 & 093224.3 & -740155 & 0.80 & 0.60 & Ir & $\mathrm{VL}$ & 16.69 & NED & 0.48 & \\
\hline 33 & 095330.2 & -675006 & 0.70 & 0.50 & Sph? & $\mathrm{L}$ & 17.2 & IK & 0.80 & \\
\hline 34 & 100311.4 & -314717 & 0.80 & 0.40 & Ir & $\mathrm{L}$ & 17.5 & IK & 0.45 & \\
\hline 35 & 100532.0 & -640716 & 1.00 & 0.90 & Ir? & $\mathrm{VL}$ & 14.3 & NED & 0.93 & \\
\hline 36 & 102825.0 & -460051 & 1.60 & 1.20 & Sph? & $\mathrm{L}$ & 14.86 & NED & 0.77 & \\
\hline 37 & 102918.9 & -215933 & 1.20 & 0.60 & Ir & $\mathrm{L}$ & 16.44 & NED & 0.28 & \\
\hline 38 & 104338.3 & -442504 & 0.80 & 0.40 & Ir & $\mathrm{L}$ & 16.8 & IK & 0.77 & \\
\hline 39 & 105300.6 & -472612 & 1.10 & 0.80 & Ir & $\mathrm{VL}$ & 17.5 & IK & 0.93 & \\
\hline 40 & 105516.8 & -475440 & 2.00 & 1.20 & Ir? & $\mathrm{VL}$ & 16.03 & NED & 0.95 & \\
\hline 41 & 111739.2 & -684849 & 0.90 & 0.60 & Ir & $\mathrm{VL}$ & 18.0 & IK & 1.20 & \\
\hline 42 & 112411.0 & -722019 & 1.00 & 0.60 & Ir? & $\mathrm{VL}$ & 17.8 & IK & 1.46 & \\
\hline 43 & 113251.7 & -411614 & 0.70 & 0.50 & Ir? & $\mathrm{VL}$ & 17.9 & IK & 0.35 & \\
\hline 44 & 113525.2 & -385637 & 1.40 & 0.90 & Ir & $\mathrm{H}$ & 15.85 & NED & 0.61 & \\
\hline 45 & 113759.9 & -465544 & 0.60 & 0.50 & Ir & $\mathrm{VL}$ & 18.2 & IK & 0.71 & \\
\hline
\end{tabular}


Table 1. b) continued.

\begin{tabular}{|c|c|c|c|c|c|c|c|c|c|c|}
\hline kks & \multicolumn{2}{|c|}{ RA (1950.0) Dec } & $a$ & $b$ & Type & S.B. & $B_{\mathrm{t}}$ & Ref.(B) & $A_{b}$ & Notes \\
\hline & $\mathrm{h} \mathrm{m} \mathrm{s}$ & & $\operatorname{arc}$ & $\min$ & & & & & & \\
\hline 1 & 2 & 3 & 4 & 5 & 6 & 7 & 8 & 9 & 10 & 11 \\
\hline 46 & 114002.3 & -253808 & 0.80 & 0.70 & Ir & $\mathrm{L}$ & 16.60 & NED & 0.20 & \\
\hline 47 & 115457.6 & -275045 & 1.30 & 1.10 & $\mathrm{Ir} / \mathrm{Sph}$ & $\mathrm{VL}$ & 17.5 & IK & 0.32 & conf. E440-35 \\
\hline 48 & 120301.0 & -432930 & 1.30 & 0.90 & $\mathrm{Ir}$ & $\mathrm{L}$ & 17.20 & NED & 0.53 & \\
\hline 49 & 120502.6 & -305458 & 0.80 & 0.60 & $\mathrm{Ir} / \mathrm{Sph}$ & $\mathrm{L}$ & 17.25 & NED & 0.28 & \\
\hline 50 & 120655.1 & -300507 & 0.60 & 0.45 & Sph? & $\mathrm{L}$ & 18.0 & IK & 0.25 & \\
\hline 51 & 124136.2 & -423958 & 0.90 & 0.40 & $\mathrm{E} / \mathrm{Sph}$ & $\mathrm{L}$ & 16.7 & IK & 0.38 & \\
\hline 52 & 125513.5 & -453231 & 1.60 & 1.10 & $\mathrm{Ir} / \mathrm{Sm}$ & $\mathrm{VL}$ & 16.4 & IK & 0.38 & \\
\hline 53 & 130824.3 & -383826 & 0.80 & 0.70 & Sph & $\mathrm{VL}$ & 17.3 & IK & 0.38 & \\
\hline 54 & 131844.5 & -313729 & 1.10 & 0.75 & Ir? & EL & 17.6 & IK & 0.29 & \\
\hline 55 & 131917.8 & -422800 & 0.80 & 0.70 & Sph & EL & 18.5 & IK & 0.63 & Centaurus. gr. \\
\hline 56 & 133256.1 & -561646 & 1.10 & 0.95 & $\mathrm{Ir} ?$ & $\mathrm{~L}$ & 16.7 & IK & 3.02 & \\
\hline 57 & 133838.5 & -421947 & 0.80 & 0.70 & Sph & EL & 18.1 & IK & 0.39 & Centaurus gr.? \\
\hline 58 & 134305.7 & -360441 & 1.00 & 0.70 & Sph? & VL & 17.1 & IK & 0.27 & \\
\hline 59 & 134443.9 & -530608 & 3.00 & 1.30 & Ir & $\mathrm{L}$ & 14.2 & NED & 2.13 & \\
\hline 60 & 135042.2 & -615918 & 1.70 & 1.20 & $\mathrm{RN} ?$ & $\mathrm{~L}$ & 17.1 & IK & 77.92 & \\
\hline 61 & 150556.6 & -674515 & 1.50 & 0.60 & Ir & $\mathrm{L}$ & 18.0 & IK & 0.79 & \\
\hline 62 & 154049.7 & -275507 & 1.00 & 0.50 & $\mathrm{Ir}$ & $\mathrm{L}$ & 18.1 & IK & 0.90 & \\
\hline 63 & 155642.3 & -310845 & $2.5:$ & 1.5: & Ir? & EL & 16.0 & IK & 0.94 & \\
\hline 64 & 160601.7 & -653714 & 0.90 & 0.75 & Ir? & $\mathrm{L}$ & 16.9 & IK & 0.56 & \\
\hline 65 & 164408.9 & -270517 & 0.80 & 0.60 & $\mathrm{~S} ?$ & $\mathrm{~L}$ & 17.5 & IK & 1.24 & \\
\hline 66 & 164929.1 & -590029 & 1.50 & 0.70 & $\mathrm{Ir}$ & $\mathrm{VL}$ & 16.7 & IK & 0.58 & comp. to NGC $6221 ?$ \\
\hline 67 & 170038.2 & -184814 & 0.70 & 0.70 & Ir & $\mathrm{VL}$ & 17.7 & IK & 1.40 & \\
\hline 68 & 181805.8 & -621742 & 1.00 & 0.55 & Ir & $\mathrm{L}$ & 16.59 & NED & 0.34 & \\
\hline 69 & 184622.8 & -640402 & 0.40 & 0.30 & Ir & $\mathrm{L}$ & 18.09 & NED & 0.37 & comp. to NGC $6744 ?$ \\
\hline 70 & 190212.3 & -640228 & 0.55 & 0.50 & Ir? & $\mathrm{L}$ & 17.7 & IK & 0.20 & comp. to NGC $6744 ?$ \\
\hline 71 & 190400.6 & -634841 & 2.70 & 0.70 & Ir & $\mathrm{L}$ & 15.10 & NED & 0.19 & comp. to NGC 6744 \\
\hline 72 & 190740.0 & -635621 & 0.60 & 0.45 & $\mathrm{Ir}$ & VL & 17.9 & IK & 0.17 & comp. to NGC $6744 ?$ \\
\hline 73 & 191720.4 & -604641 & 1.20 & 1.00 & Ir & $\mathrm{L}$ & 16.9 & IK & 0.23 & \\
\hline 74 & 192316.9 & -610643 & 0.90 & 0.45 & $\mathrm{Ir}$ & $\mathrm{VL}$ & 17.6 & IK & 0.34 & \\
\hline 75 & 201721.2 & -220348 & 0.50 & 0.45 & $\mathrm{Ir}$ & $\mathrm{L}$ & 18.2 & IK & 0.34 & \\
\hline 76 & 203957.8 & -612652 & 0.80 & 0.70 & Ir & $\mathrm{L}$ & 17.11 & NED & 0.23 & \\
\hline 77 & 204944.9 & -390643 & 0.80 & 0.30 & Ir & $\mathrm{L}$ & 17.8 & IK & 0.24 & \\
\hline 78 & 215030.9 & -264854 & 1.10 & 0.50 & Ir & $\mathrm{L}$ & 16.25 & NED & 0.15 & \\
\hline 79 & 220402.0 & -463931 & 0.9: & $0.7:$ & Sph? & $\mathrm{L}$ & 17.5 & IK & 0.06 & \\
\hline 80 & 221251.8 & -594648 & 0.90 & 0.70 & Ir & $\mathrm{L}$ & 17.4 & IK & 0.11 & \\
\hline 81 & 224429.4 & -190633 & 1.10 & 0.60 & Ir & $\mathrm{L}$ & 16.47 & NED & 0.14 & \\
\hline
\end{tabular}

from high surface brightness galaxies (spiral galaxies of late type) of 6 to $7 M_{\odot} / \mathrm{pc}^{2}$ with a value of $\sim 3.2 M_{\odot} / \mathrm{pc}^{2}$ for LSB galaxies. For LSB galaxies a trend of decreasing H I surface density with decreasing surface brightness has been observed (e.g. van de Hulst et al. 1993). We do confirm that the $\mathrm{H}$ I surface density decreases with decreasing surface brightness, which seems to suggest a correlation with star formation rate. On the other hand the galaxies in our sample are so small that their size and luminosity is outside the range of galaxies studied by Kennicutt (1998), which complicates a direct comparison.

\section{Conclusion}

In this paper we present Hi observations of two other lists of the Karachentsev catalog of candidates for nearby dwarf galaxies. The detection rate for these 165 galaxies is $48 \%$. Including Papers I to III we have now performed H I observations of all the published Karachentsev dwarf galaxies. We discuss the whole sample of 601 galaxies and confirm the dwarfish character of these galaxies by the shape and linewidths $\left(\left\langle w_{50}\right\rangle=51 \mathrm{~km} \mathrm{~s}^{-1}\right)$ of the Hi profiles, their linear sizes $\left(\left\langle A_{0}\right\rangle=3.2 \mathrm{kpc}\right)$ 
Table 1. c) List of new dwarf galaxy candidates from the kk list.

\begin{tabular}{|c|c|c|c|c|c|c|c|c|c|c|}
\hline $\mathrm{kk}$ & \multicolumn{2}{|c|}{ RA (1950.0) Dec } & $a$ & $b$ & Type & S.B. & $B_{\mathrm{t}}$ & Ref.(B) & $A_{b}$ & Notes \\
\hline & $\mathrm{h} \mathrm{m} \mathrm{s}$ & & $\operatorname{arcmi}$ & & & & & & & \\
\hline 1 & 2 & 3 & 4 & 5 & 6 & 7 & 8 & 9 & 10 & 11 \\
\hline 11 & 010603.4 & -382835 & 0.9 & 0.4 & $\operatorname{Im}$ & $\mathrm{H}$ & & & & K3, AM0106-382 distant? \\
\hline 47 & 052749.0 & -873736 & 1.0 & 0.45 & $\operatorname{Im}$ & $\mathrm{L}$ & 17.78 & NED & 0.652 & AM 0528-873 \\
\hline 63 & 073720.0 & -691338 & 1.5 & 0.9 & Sph & VL & & & & FG219, AM0737-691 \\
\hline 87 & 101237.6 & -443608 & 1.2 & 1.0 & Sm? & $\mathrm{H}$ & 13.49 & NED & 0.902 & AM 1012-443 \\
\hline 88 & 101357.4 & -394423 & 0.9 & 0.5 & Ir & VL & 16.7 & IK & 0.592 & AM 1013-394 \\
\hline 179 & 130040.5 & -461907 & 1.2: & 0.9: & Sph & $\mathrm{L}$ & & & & FG367, AM1300-461 \\
\hline 272 & 130333.1 & -493338 & 1.6 & 0.6 & Ir & $\mathrm{L}$ & 17.83 & NED & 0.822 & ESO 219-G027 \\
\hline 184 & 130615.0 & -504446 & 1.9 & 0.4 & Ir & $\mathrm{H}$ & & & & AM1305-501 distant? \\
\hline 189 & 130953.2 & -413401 & 1.0 & 0.6 & Sph? & VL & & & & $\mathrm{EL}$ in $\mathrm{R}$ \\
\hline 190 & 131014.6 & -443728 & 2.7 & 1.7 & Sph & $\mathrm{L}$ & & & & FG373, AM1310-443 \\
\hline 197 & 131906.8 & -421620 & 1.5 & 1.1 & Sph & $\mathrm{VL}$ & & & & near NGC 5128 \\
\hline 203 & 132429.6 & -450536 & 0.5 & 0.5 & $\mathrm{Ir} / \mathrm{Sph}$ & $\mathrm{L}$ & & & & AM1324-450 \\
\hline 211 & 133903.4 & -445711 & 1.2 & 1.1 & Sph? & $\mathrm{L}$ & & & & AM1339-445 \\
\hline 213 & 134034.6 & -433104 & 0.6 & 0.3 & Sph? & VL & & & & $\mathrm{EL}$ in $\mathrm{R}$ \\
\hline 214 & 134044.0 & -452941 & 0.75 & 0.4 & $\mathrm{Ir}$ & $\mathrm{L}$ & & & & AM1340-453 distant? \\
\hline 217 & 134313.4 & -452606 & 1.0 & 0.9 & Sph & $\mathrm{L}$ & & & & AM1343-452 \\
\hline 221 & 134540.5 & -464454 & 1.5: & 1.0: & $\mathrm{Sph}$ & EL & & & & near br.star \\
\hline 222 & 134604.9 & -475721 & 0.5 & 0.4 & $\mathrm{Ir} / \mathrm{Sph}$ & $\mathrm{L}$ & & & & AM1346-475 \\
\hline 226 & 135301.2 & -452447 & 1.1 & 0.7 & $\mathrm{Ir}$ & $\mathrm{L}$ & 15.73 & NED & 0.311 & \\
\hline 235 & 150311.2 & -394236 & 0.8 & 0.6 & $\mathrm{Ir}$ & $\mathrm{L}$ & & & & FG438 distant? \\
\hline 239 & 152533.2 & -423636 & 1.9 & 0.7 & $\mathrm{Ir}$ & $\mathrm{L}$ & 15.06 & NED & 0.621 & FG444 \\
\hline 244 & 190920.3 & -615957 & 0.45 & 0.45 & Sph? & $\mathrm{L}$ & 17.0: & IK & 0.239 & AM1909-615 \\
\hline 248 & 202246.0 & -713423 & 0.8 & 0.4 & Ir & $\mathrm{L}$ & 15.87 & NED & 0.208 & FG499 distant? \\
\hline 256 & 220904.6 & -432529 & 0.6 & 0.5 & $\operatorname{Im}$ & $\mathrm{L}$ & 16.89 & NED & 0.053 & AM 2209-432 \\
\hline 260 & 231146.9 & -435239 & 4.5 & 1.8 & Ir & VL & 15.0: & IK & 0.049 & \\
\hline
\end{tabular}

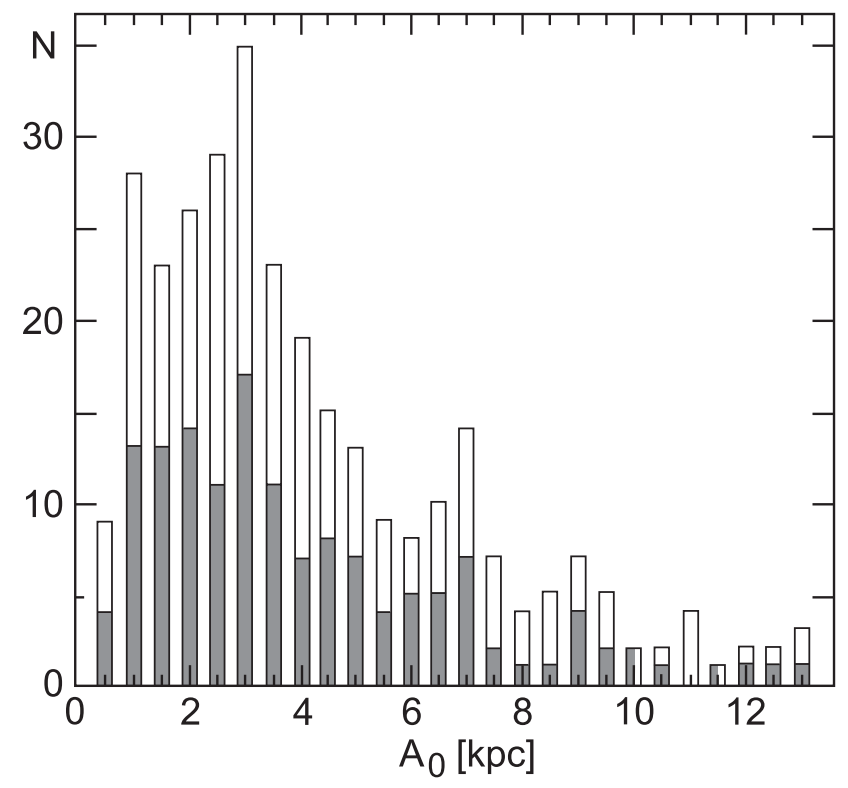

Fig. 6. The distribution of the linear blue diameters $A_{0}[\mathrm{kpc}]$ binned to $0.5 \mathrm{kpc}$ of all galaxies (Papers I to IV) and the detected galaxies of Paper I (in grey) is shown. There are seven galaxies with linear diameters $A_{0}$ between 13 and $17 \mathrm{kpc}$ beyond the scope of this figure. and absolute magnitudes $\left(\left\langle M_{B T}^{0}\right\rangle=-14.8\right)$. Histograms of Figs. 3 to 6 demonstrate that subsamples of the Karachentsev catalog show the general trends of the whole catalog. The indicative H I surface density of these low surface brightness galaxies in our sample is definitely lower than for high surface brightness galaxies and decreases with decreasing surface brightness.

Acknowledgements. We have made extensive use of the NASA/IPAC Extragalactic Database (NED, which is operated by the Jet Propulsion Laboratory, Caltech, under contract with the National Aeronautics and Space Administration), and the Digitized Sky Survey (DSS-1) produced at the Space Telescope Science Institute under U.S. Goverment grant NAG W-2166. The Parkes telescope is part of the Australia Telescope which is founded by the Commonwealth of Australia for operation as a National Facility by CSIRO. Our project is supported by DFG grant No. 436 RUS 113/470/0.

\section{References}

Banks, G. D., Disney, M. J., Knezek, P. M., et al. 1999, ApJ, 524,612

Barnes, D. G., Staveley-Smith, L., \& de Blok, W. J. G. 2001, MNRAS, 322, 486 
de Blok, W. J. G. 1997, Ph.D. Thesis Univ. of Groningen

Eder, J. A., \& Schombert, J. 2000, ApJS, 131, 47

Henning, P. A., Kraan-Korteweg, R. C., Rivers, A. J., et al. 1998, AJ, 115, 584

Huchtmeier, W. K., \& Richter, O. G. 1988, A\&A, 203, 237

Huchtmeier, W. K., \& Richter, O. G. 1989, A\&A, 210, 1

Huchtmeier, W. K., Karachentsev, I. D., Karachentseva, V. E., \& Ehle, M. H. 2000a, A\&AS, 141, 469 (Paper I)

Huchtmeier, W. K., Karachentsev, I. D., \& Karachentseva, V. E. 2000b, A\&AS, 147, 187 (Paper II)

Karachentsev, I. D. 1994, A\&A, Trans. 6, 1

Karachentsev, I. D., Karachentseva, V. E., \& Huchtmeier, W. K. 2001, A\&A, 366, 428 (Paper III)

Karachentsev, I. D., Karachentseva, V. E., Suchkov, A. A., \& Grebel, E. K. 2000, A\&AS, 145, 415
Karachentsev, I. D., \& Makarov, D. I. 1996, AJ, 111, 535 A\&AS, 135, 221

Karachentseva, V. E., \& Karachentsev, I. D. 2000, A\&AS, 146, 359

Kennicutt, R. 1998, ApJ, 498, 541

Kilborn, V., Webster, R., \& Staveley-Smith, L. 1999, PASA, 16, 8 (HIPASS)

Kraan-Korteweg, R. C., \& Tammann, G. A. 1979, Astron. Nachr., 300, 181

Salpeter, E., \& Hoffman, G. 1996, ApJ, 465, 595

Schlegel, D. J., Finkbeiner, D. P., \& Davis, M. 1998, ApJ, 500, 525

van de Hulst, J. M., Skillman, E. D., Smith, T. R., et al. 1993, AJ, 106,548 